\title{
Improving the Surface Properties of Cp-Ti by Pulsed Electro-Spark Deposition
}

\author{
M.S. YILMAZ ${ }^{a, *}$, E. ATAR ${ }^{a}$, O. ŞAHIN ${ }^{a}$ AND E.S. KAYALI ${ }^{b}$ \\ ${ }^{a}$ Gebze Institute of Technology, Department of Materials Science and Engineering, 41400 Gebze, Kocaeli, Turkey \\ ${ }^{b}$ Istanbul Technical University, Department of Metallurgy and Materials Engineering \\ 34469 Maslak, Istanbul, Turkey
}

\begin{abstract}
$\mathrm{Al}-\mathrm{Ti}$ intermetallic compounds were coated by electro-spark deposition. Commercially pure titanium $(\mathrm{Cp}-\mathrm{Ti})$ (grade 2) and aluminum rods were used as substrate and electrode materials, respectively. During the electro-spark deposition experiments the following pulse parameters in a group were used: pulse current amplitude, pulse duration and pause between the pulses of a pulse group with 100-300-500 A, 50-100 $\mu \mathrm{s}$, and $100 \mu \mathrm{s}$, respectively. $\mathrm{Al}-\mathrm{Ti}$ coatings having a thickness range of $15-30 \mu \mathrm{m}$ were formed on the surface of titanium substrate using electro-spark deposition process. The coating properties such as the roughness and the thickness increased with increasing pulse duration and pulse current amplitude. The mass transfer coefficient decreased with increasing pulse current amplitude and pulse duration. The quality of the adhesion characters of the coatings, determined by Rockwell-C adhesion test, becomes worse from HF-2 to HF-4, due to increase of pulse current amplitude. Depending on the electro-spark deposition process parameters, the coating formed as AlTi or as layered structure consisting of $\mathrm{AlTi}+\mathrm{AlTi}_{3}$ when pulse current amplitude increases. The $\mathrm{Al}-\mathrm{Ti}$ coatings include some micro cracks lying throughout the coating from surface to interface which is nature of this process. The maximum cross-sectional hardness of the coatings were in the range of $800-1150 \mathrm{HV}$.
\end{abstract}

DOI: $10.12693 /$ APhysPolA.125.593

PACS: 68.47.De, 61.82.Bg

\section{Introduction}

Titanium alloys are the most attractive metallic materials in recent years due to their high corrosion resistance, low density, low elastic modulus, biocompatibility, and high strength. Due to its suitability in aerospace, medical, and dental applications, the interests and studies on titanium and its alloys have increased. Although, titanium and its alloys show better mechanical and chemical properties comparing to some other metals, they have weakness when used in frictional applications [1, 2]. Because of this weakness, the aim of this study is to improve surface properties of $\mathrm{CpTi}$, such as coating with $\mathrm{Ti}-\mathrm{Al}$ intermetallics with electro-spark deposition (ESD) process. $\mathrm{Al}-\mathrm{Ti}$ intermetallics coating will give attractive chemical and mechanical properties to titanium-based structural materials [3].

ESD treatment provides several benefits such as: strong metallurgical bond with the substrate, wear proof, easy to use, low cost for surface engineering and small heat affected zone on the work-piece. ESD equipment can provide coatings with a better surface quality and interface (coating-substrate) characteristics, and opportunities such as faster and more efficient production. There are various parameters affecting the properties of coatings obtained by ESD method, such as electrical parameters (voltage, capacitance, ampere, inductance, pulse

*corresponding author; e-mail: msyilmaz@gyte.edu.tr intensity and duration), working medium (gas medium, temperature), electrode material (composition, density, shape, travelling speed, contact intensity) and substrate material (material choice, surface preparation, cleaning, temperature, shape) [4].

\section{Experimental}

Commercial ESD machine equipped with a hand-held gun was used. Commercially pure titanium substrates (cathode) and commercially pure aluminum rods as electrode (anode) were used in this study. Parameters of ESD process were investigated experimentally and literarily, in order to form a better coating layer on the substrates. All substrates were mechanically ground with 200-1200 mesh emery papers and cleaned ultrasonically for $5 \mathrm{~min}$ with acetone.

During the experiments the following pulse parameters in a group were used: pulse current amplitude, pulse duration and pause between the pulses of a pulse group with 100-300-500 A, 50-100 $\mu \mathrm{s}$, and $100 \mu \mathrm{s}$, respectively. Accordingly, electrical charge $(2000 \mathrm{mC})$, system voltage $(17 \mathrm{~V})$, coverage rate $\left(60 \mathrm{~s} / \mathrm{cm}^{2}\right)$, rectangle pulse patterns, and vibration frequency of the electrode holder were kept constant.

In the present research, 200 Metler Toledo Precision Balance, which has $0.001 \mathrm{mg}$ sensitivity, was used to determine the mass transformation. The surface roughness measurement was performed by using Perthometer S8P (Perthen-Mahr, Göttingen, Germany) optical profilometer. Rockwell-C test was used to analyze the adhesion properties of the coatings. Identification of the 
phases was performed by using a Philips PW 3710 type $\mathrm{X}$-ray diffractometer. X-ray diffraction (XRD) analyses were conducted by using $\mathrm{Cu} K_{\alpha}$ radiation tube. Diffraction angle was between 20 and $90^{\circ}$, with a step increment of $0.02^{\circ}$ and account time of $1 \mathrm{~s}$. Microstructures of the coatings were investigated by computer equipped Philips XL30 type field emission scanning electron microscopy (SEM) equipped with energy dispersive X-ray spectroscopy (EDX). Cross-sectional hardness tests were carried out with Mitutoyo MicroWizhard micro-hardness tester under indentation load of $5 \mathrm{~g}$.

\section{Results and discussions}

The ESD process parameters and some of the properties such as surface roughness, thickness, and mass transfer coefficient of the coatings were given in Table.

The process parameters, the code of samples and the results of some experiments

TABLE (surface roughness, thickness, and mass transfer coefficient of the coatings).

\begin{tabular}{c|c|c|c|c|c|c}
\hline \hline Pulse duration $[\mu \mathrm{s}]$ & \multicolumn{3}{|c|}{50} & \multicolumn{2}{c}{100} \\
\hline pulse currency $[\mathrm{A}]$ & 100 & 300 & 500 & 100 & 300 & 500 \\
\hline sample code & $\mathrm{A}$ & $\mathrm{B}$ & $\mathrm{C}$ & $\mathrm{D}$ & $\mathrm{E}$ & $\mathrm{F}$ \\
\hline mass loss of anode $[\mathrm{mg}]$ & 3.1 & 3.0 & 3.5 & 4.0 & 4.8 & 4.9 \\
mass gain of cathode $[\mathrm{mg}]$ & 2.7 & 1.8 & 1.5 & 2.1 & 1.5 & 0.8 \\
mass transfer coefficient & 0.87 & 0.6 & 0.43 & 0.52 & 0.31 & 0.16 \\
surface roughness $\left[R_{\mathrm{a}}, \mu \mathrm{m}\right]$ & $4.93 \pm 1.33$ & $6.56 \pm 1.48$ & $7.63 \pm 2.52$ & $6.01 \pm 1.58$ & $7.85 \pm 1.14$ & $8.21 \pm 1.35$ \\
coating thickness $[\mu \mathrm{m}]$ & $15.83 \pm 0.79$ & $16.67 \pm 1.02$ & $17.31 \pm 0.64$ & $21.28 \pm 3.29$ & $25.00 \pm 2.52$ & $24.36 \pm 2.69$
\end{tabular}

In Table it was noticed that the mass loss of anode increases with increasing pulse current amplitude and pulse duration, however the mass gain of cathode decreased with increasing pulse current amplitude and pulse duration (Fig. 1a). The coating properties such as roughness (Fig. 1b) and thickness (Fig. 1c) increased with increasing pulse duration and pulse current amplitude. Intermetallic coatings having thickness of $15-$ $30 \mu \mathrm{m}$ were formed on the surface of titanium substrate using the ESD.

Although there is a decrease in mass transfer coefficient with increasing pulse current amplitude and pulse duration, still the coating thicknesses generally increased, which shows that the increase in coating thickness is independent of the mass transfer coefficient. Diffusion mechanism becomes more active by the increase on pulse current amplitude and pulse duration.

Rockwell-C adhesion test was used to analyze the adhesion properties of the coatings qualitatively. In this test, we analyzed the cracks and the breaks of the coatings. The characteristics of the coatings compared with the standard Rockwell-C adhesion test and defined for sufficient adhesion as HF 1 to HF 4 and for insufficient adhesion as HF 5 and HF 6 [5]. Examination of the Rockwell-C indents showed that there were only cracks on the coatings, where no coating detachment was observed (Fig. 2). Thus, the adhesion of the coatings are HF-2 quality for sample-A and sample-D, HF-3 quality for sample-B and sample-E and HF-4 quality for sample- $\mathrm{C}$ and sample-F as seen in Fig. 2. The quality of the adhesion characters of the samples becomes better, due to decrease of the pulse current amplitude.

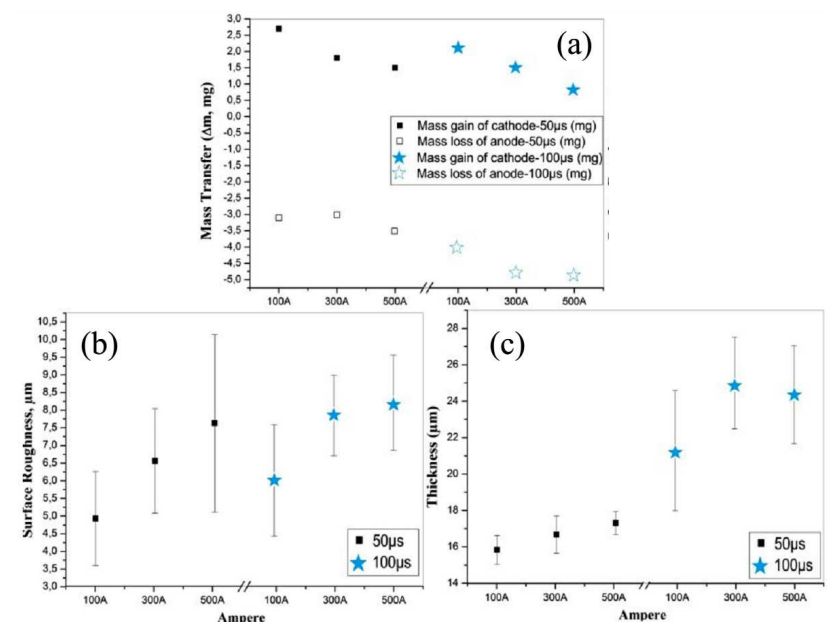

Fig. 1. The mass transfer (a), surface roughness (b), and coating thickness (c) of samples, with the change of pulse duration and pulse current amplitude.

\subsection{XRD and SEM analysis}

XRD patterns of ESD coatings are illustrated in Fig. 3. The peaks of AlTi, $\mathrm{AlTi}_{3}, \mathrm{AlTi}-\mathrm{O}_{2}$ and $\mathrm{TiN}$ phases were identified on the XRD patterns. It should be also noticed that peaks of AlTi phase were determined on the XRD patterns of all coated specimens. While the peak intensities of AlTi phase decreased with increase of the pulse current amplitude (300-500 A), the peak intensities of $\mathrm{AlTi}_{3}$ phase increased with the increase of the pulse current amplitude. 

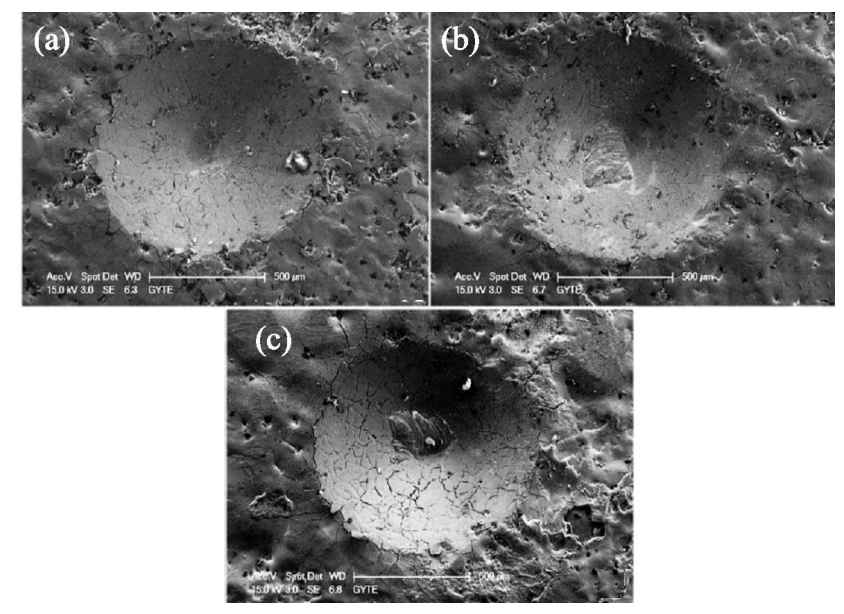

Fig. 2. Micrographs of Rockwell-C indentation tests: (a) HF2-sample A, (b) HF3-sample B, (c) HF4-sample C.

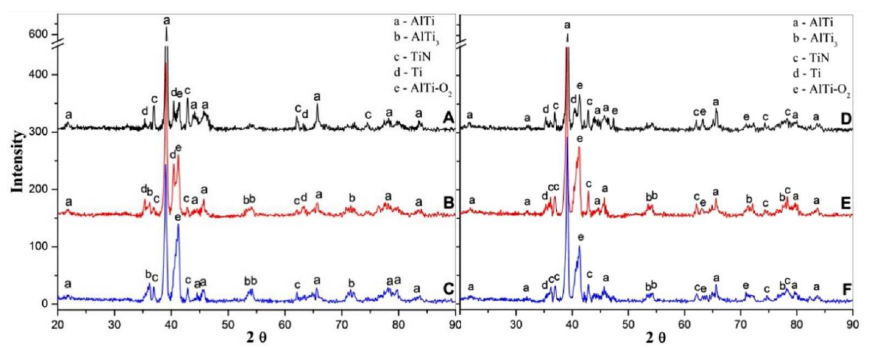

Fig. 3. XRD pattern of ESD coatings.

Cross-sectional SEM micrographs of coatings showed that there were rather homogeneous mixtures of coating layers on specimens when pulse current amplitude was 100 A (Fig. 4a-c, sample-A,D). On the other hand, the coatings become laminar when the pulse current amplitudes are 300 and $500 \mathrm{~A}$ (Fig. 4b).
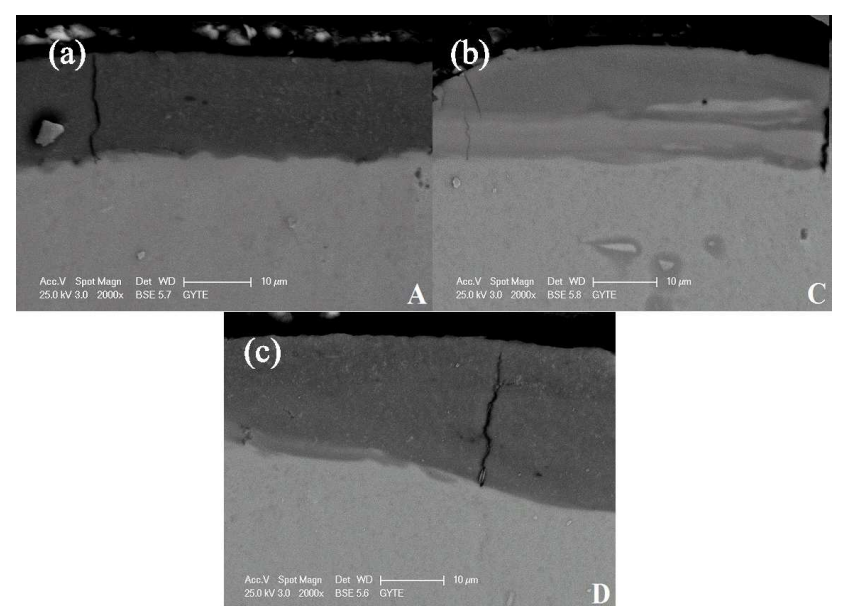

Fig. 4. SEM micrographs of sample-A (a), sample-C (b) and sample-D (c).
Additionally, SEM analysis showed microcracks lying throughout the coating from surface to interface (Fig. 4). Variations on process parameters did not cause significant changes on amount and size of these cracks. These microcracks were evaluated as the nature of this process $[3,6]$. These cracks are generated by the combination effect of cooling rate and thermal expansion differences [6].

These microcracks are seen in all studies carried on using these material couples. Due to prevent crack formation heating of the substrate material before coating process and controlled cooling or repairing cracks by laser melting treatment after coating process was performed $[4,7-10]$.
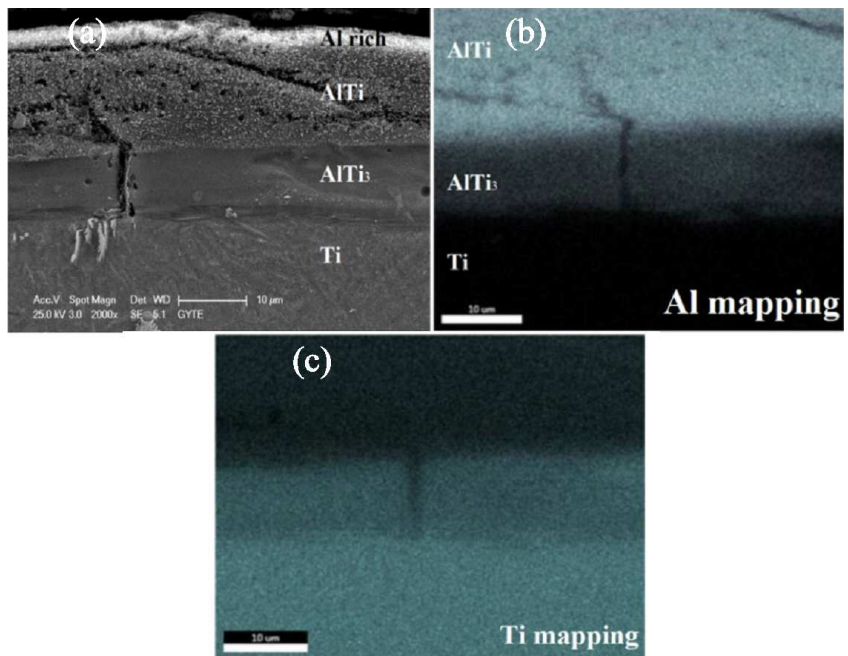

Fig. 5. SEM micrographs of etching and elemental mapping of sample-B.

Homogeneous and lamellar coating layers were formed during ESD experiments. The coating layers are illustrated in Fig. 5a. A very thin layer of Al rich phase exists on the surface of the coating. Toward to interior, the layers of AlTi and $\mathrm{AlTi}_{3}$ phases were observed. These layers were clearly seen after the etching of the sample-B (Fig. 5a). The elemental aluminum (Fig. 5b) and titanium (Fig. 5c) EDX mapping results support the presence of AlTi and $\mathrm{AlTi}_{3}$ phases.

The structural change from homogeneous to lamellar coating occurred due to the change of pulse current amplitude. This behavior may be related to high pulse current amplitude which causes the temperature rises. This temperature rise allows to reach high diffusion rate of aluminum in the coating which results lamellar as AlTi and $\mathrm{AlTi}_{3}$ from surface to substrate [3].

\subsection{Hardness}

The cross-sectional hardness profiles of three different samples are given in Fig. 6. The maximum cross-sectional hardness of the coatings were in the range of 800-1150 HV. These hardness values are much higher than that of $\mathrm{Cp}-\mathrm{Ti}$ substrate hardness $(230 \mathrm{HV})$. 


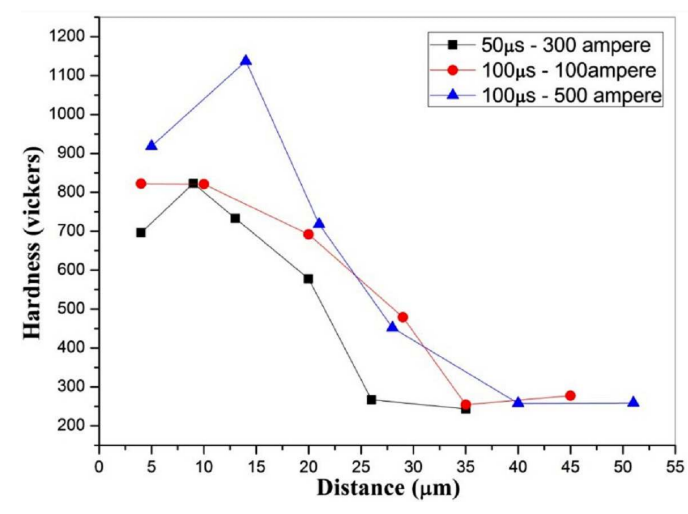

Fig. 6. Vickers hardness profiles of the coating layers.

The hardness values of this study are up to three times higher than the values reported in the literature. Annealed titanium aluminides hardnesses are around: $250 \mathrm{HV}$ for $\mathrm{Ti}-25 \mathrm{Al}, 290 \mathrm{HV}$ for $\mathrm{Ti}-48 \mathrm{Al}$ ( $\gamma$-phase), $370 \mathrm{HV}$ for $\mathrm{Ti}-55 \mathrm{Al}$ ( $\gamma$-phase) [3, 11]. This high hardness values are probably due to the rapid cooling of the ESD process. The increase in hardness provides excellent wear resistance of this intermetallic coatings [3].

\section{Conclusion}

In this study, the following conclusions were obtained.

Titanium aluminum coating layers having a thickness range of $15-30 \mu \mathrm{m}$ were formed on the surface of titanium substrate using ESD process. The coating properties such as the roughness and the thickness increased with increasing pulse duration and pulse current amplitude. The mass transfer coefficient decreased with increasing pulse current amplitude and pulse duration. The quality of the adhesion characters of the samples, determined by Rockwell-C adhesion test, becomes worse from HF-2 to HF-4, due to increase of pulse current amplitude. Depending on the ESD process parameters, the coating formed as $\mathrm{TiAl}$ or as layered structure consisting of $\mathrm{TiAl}+\mathrm{Ti}_{3} \mathrm{Al}$ when pulse current amplitude increases. The titanium aluminum intermetallic coatings include some microcracks lying throughout the coating from surface to interface which is nature of this process. The maximum cross-sectional hardness of the coatings were in the range of 800-1150 $\mathrm{HV}$.

\section{Acknowledgments}

The authors express their thanks to technicians Adem Sen and Ahmet Nazım for their kind assistance during XRD and SEM experimental studies.

\section{References}

[1] C.B. Tang, Appl. Surf. Sci. 257, 6364 (2011).

[2] E. Atar, E.S. Kayali, H. Cimenoglu, Surf. Coat. Technol. 202, 4583 (2008).

[3] N.M.K. Mizuta, S. Kirihara, Y. Miyamoto, Mater. Sci. Eng. A 492, 199 (2008).

[4] R.N. Johnson, in: 45th Annual Technical Conf. Proc., Ed. R.N. Johnson, Pacific Nothwest National Laboratory, Richland (WA) 2002, p. 87.

[5] W. Heinke, A.L.A. Matthews, G. Berg, C. Friedrich, E. Broszeit, Thin Solid Films 270, 431 (1995).

[6] M.S. Yllmaz, M.Sc. Thesis, İstanbul Teknik Üniversitesi, 2012.

[7] A.V. Ribalko, O. Sahin, Surf. Coat. Technol. 201, 1724 (2006).

[8] A.V. Ribalko, O. Sahin, Metal Working 16, No. 4 (2003) (in Russian).

[9] K.H.İ.B. Korkmaz, Metall. Mater. 48, 153 (2010).

[10] F.J.D.H.P. Appel, M. Oehring, Gamma Titanium Aluminide Alloys, Science and Technology, Weinheim, Germany 2011.

[11] M. Schloffer, Intermetallics 22, 231 (2011). 